\title{
A thermal orbital-free density functional approach
}

Cite as: J. Chem. Phys. 151, 014103 (2019); https://doi.org/10.1063/1.5100231

Submitted: 16 April 2019 . Accepted: 06 June 2019. Published Online: 02 July 2019

Á. Nagy (iD)

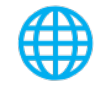

View Online

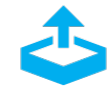

Export Citation

\section{ARTICLES YOU MAY BE INTERESTED IN}

An alternative derivation of orbital-free density functional theory

The Journal of Chemical Physics 150, 204109 (2019); https://doi.org/10.1063/1.5096405

The first order atomic fragment approach-An orbital-free implementation of density

functional theory

The Journal of Chemical Physics 151, 024109 (2019); https://doi.org/10.1063/1.5099217

Implementation of analytic gradients for CCSD and EOM-CCSD using Cholesky

decomposition of the electron-repulsion integrals and their derivatives: Theory and

benchmarks

The Journal of Chemical Physics 151, 014110 (2019); https://doi.org/10.1063/1.5100022

The Journal

of Chemical Physics

The Emerging Investigators Special Collection and Awards Recognizing the excellent work of early career researchers! 


\title{
A thermal orbital-free density functional approach
}

\author{
Cite as: J. Chem. Phys. 151, 014103 (2019); doi: 10.1063/1.5100231 \\ Submitted: 16 April 2019 - Accepted: 6 June 2019 • \\ Published Online: 2 July 2019
}

Á. Nagy

\begin{abstract}
AFFILIATIONS
Department of Theoretical Physics, University of Debrecen, H-4002 Debrecen, Hungary
\end{abstract}

${ }^{a)}$ E-mail: anagy@phys.unideb.hu

\begin{abstract}
A generating function $\sigma$ is defined for spherically symmetric systems. Compared to the density, the generating functional has two extra variables and reduces to the density if these variables are equal to zero. It is proved that $\sigma$ satisfies a differential equation that contains only the derivatives of $\sigma$ and the Kohn-Sham potential. A Schrödinger-like equation for the square root of $\sigma$ is also derived. The effective potential of this equation is the sum of the Kohn-Sham potential and a term that is expressed with an integral containing the derivatives of $\sigma$. The noninteracting kinetic energy can be calculated in the knowledge of $\sigma$. The theory is valid in case of zero and nonzero temperatures as well. For nonspherically symmetric systems, the muffin-tin approximation can be applied.
\end{abstract}

Published under license by AIP Publishing. https://doi.org/10.1063/1.5100231

\section{INTRODUCTION}

Density functional theory (DFT) is one of the most beneficial and effective methods for examining the electron structure. The original Hohenberg-Kohn theory ${ }^{1}$ was extended to nonzero temperature by Mermin. ${ }^{2}$ In principle, the Euler equation can be used to obtain the density. But the kinetic energy functional is unknown. Therefore, Kohn and Sham (KS) ${ }^{3}$ introduced a noninteracting system in which the particles move independently in a common, local potential field. This Kohn-Sham (KS) potential is defined by the constraint that the density of the noninteracting system is the same as that of the true, interacting system. The kinetic energy can be expressed with the one-particle orbitals and the variational principle leads to the KS equations.

Nowadays, the great majority of DFT calculations are done by solving the KS equations. For a system with many particles, there are a lot of occupied KS orbitals. Therefore, it is reasonable to go back to the original Euler equation and seek to find appropriate approximation for the kinetic energy functional. It is especially essential in case of nonzero temperature because there are a huge number of partially occupied KS orbitals even for a one-particle system. This explains the increasing efforts done in the direction of an orbital-free DFT. ${ }^{4-}$
In this paper, a different approach is presented. Here, the need for the kinetic energy functional is avoided. Instead, a generating function $\sigma$ is defined. It has two extra variables compared to the density and it reduces to the density if these variables are equal to zero. It is shown that $\sigma$ satisfies a differential equation that contains only the derivatives of $\sigma$ and the KS potential. Moreover, a Schrödinger-like equation for the square root of $\sigma$ is also derived. The effective potential of this equation is the sum of the Kohn-Sham potential and a term that is expressed with an integral containing the derivatives of $\sigma$. The present approach is an extension of the author's earlier method developed for zero temperature. ${ }^{13-15,17}$ Here, the notation "generating function" is used instead of the "generalized density" of the previous work ${ }^{16}$ because it shows better that this is an auxiliary quantity introduced in order to derive an orbital-free approach. The generating function has physical meaning only if the two extra variables are zero, and in that case it coincides with the density.

The present theory is valid in case of zero and nonzero temperature, as well. But it can be used for spherically symmetric systems. For nonspherically symmetric systems, the muffin-tin approximation can be applied.

The paper is organized as follows: In Sec. II, the finite temperature DFT is summarized. In Sec. III, a third-order differential 
equation for $\sigma$ and a Schrödinger-like equation for the square root of $\sigma$ are derived. Section IV is devoted to discussion and an illustrative example.

\section{FINITE TEMPERATURE DFT}

Summarize first the extension of DFT to nonzero temperature (for details, see, e.g., Refs. 2, 3, 18, and 19). It is convenient to utilize the grand canonical ensemble. The grand canonical potential is

$$
\Omega_{\beta}[v-\mu]=\min _{n}\left\{\left\{F_{\beta}[n]+\int n(\mathbf{r})(v(\mathbf{r})-\mu)\right\},\right.
$$

where

$$
F_{\beta}[n]=\min _{\hat{\Gamma} \rightarrow n} \operatorname{tr}\left\{\hat{\Gamma}\left(\hat{T}+\hat{V}_{e e}+\frac{1}{\beta} \ln \hat{\Gamma}\right)\right\} .
$$

$\hat{T}$ and $\hat{V}_{e e}$ stand for the kinetic energy and the electron-electron energy operators, respectively. $\beta$ is the inverse temperature. The functional $F$ is defined by constrained search: the minimum is over all density matrices $\hat{\Gamma}$ that yield the given density $n . F$ is universal, that is, it depends neither on the external potential $v$ nor on the chemical potential $\mu$.

The density matrices $\Gamma$ that yield $n(\mathbf{r})$ are composed of states with different numbers of particles from 1 to infinity. This means that $n(\mathbf{r})$ corresponds to a fractional average number of particles. We can call it 'grand-canonical ensemble representable' density.

We mention in passing that one can use the canonical ensemble instead of the grand-canonical ensemble. Then, the number of particles is fixed and instead of the grand potential, the free energy is used. One can also define the functional $F_{\beta}[n]$ in the canonical ensemble and then $n(\mathbf{r})$ corresponds to the fixed number of particles. There is an inequality between the functionals in the different ensembles.

The noninteracting system can also be constructed. The KohnSham functional $F_{s, \beta}$ is defined as

$$
F_{s, \beta}[n]=\min _{\hat{\Gamma} \rightarrow n} \operatorname{tr}\left\{\hat{\Gamma}\left(\hat{T}+\frac{1}{\beta} \ln \hat{\Gamma}\right)\right\} .
$$

The Kohn-Sham equations at nonzero temperature take the form

$$
\left[-\frac{1}{2} \nabla^{2}+v_{K S}(\mathbf{r})\right] \phi_{j}(\mathbf{r})=\varepsilon_{j} \phi_{j}(\mathbf{r})
$$

where

$$
n(\mathbf{r})=\sum_{j} f_{j}\left|\phi_{j}(\mathbf{r})\right|^{2}
$$

and

$$
f_{j}=\left(1+e^{\beta\left(\varepsilon_{j}-\mu\right)}\right)^{-1} .
$$

$\phi_{j}$ and $\varepsilon_{j}$ are the orbitals and the orbital energies, respectively. The Kohn-Sham potential $v_{K S}$ can be written as the sum of the external, the classical Coulomb, and the exchange-correlation terms:

$$
v_{K S}(\mathbf{r})=v(\mathbf{r})+v_{J}(\mathbf{r})+v_{x c}(\mathbf{r}) .
$$

One can introduce the Weizsäcker kinetic energy

$$
T_{w}=\frac{1}{8} \int \frac{|\nabla n|^{2}}{n} d \mathbf{r}
$$

and define a quantity

$$
T_{\theta}=T_{s}-T_{w}
$$

and its functional derivative

$$
v_{\theta}=\frac{\delta T_{\theta}}{\delta n} .
$$

Then, the Euler equation ${ }^{11}$

$$
\frac{\delta T_{s}}{\delta n}-\frac{1}{\beta} \frac{\delta S_{s}}{\delta n}+v_{K S}=\mu
$$

takes the form

$$
\left[-\frac{1}{2} \nabla^{2}+v_{\theta}-\frac{1}{\beta} \frac{\delta S_{s}}{\delta n}+v_{K S}\right] n^{1 / 2}=\mu n^{1 / 2} .
$$

In contrast, at zero temperature, the Euler equation can be rewritten as

$$
\left[-\frac{1}{2} \nabla^{2}+v_{P}+v_{K S}\right] n^{1 / 2}=\mu n^{1 / 2},
$$

where $v_{P}$ is called the Pauli potential. ${ }^{20-24}$ Comparing Eqs. (12) and (13), we see that at finite temperature, the term $v_{\theta}$ corresponds to the Pauli potential $v_{P}$ of zero temperature. Equation (12) has an extra term containing the functional derivative of the noninteracting entropy $S_{s}$ that disappears at zero temperature and cannot be found in Eq. (13).

\section{GENERATING FUNCTION FOR SPHERICALLY SYMMETRIC SYSTEMS}

Consider a spherically symmetric system and write the KohnSham equations as

$$
-\frac{1}{2} \frac{d^{2} P_{i}(r)}{d r^{2}}+\frac{l_{i}\left(l_{i}+1\right)}{2 r^{2}} P_{i}(r)+v_{K S}(r) P_{i}(r)=\varepsilon_{i} P_{i}(r),
$$

where $P_{i}(r)$ is the radial part of the KS orbital $\phi_{j}(\mathbf{r})$ in Eq. (4) and $l_{i}$ are the azimuthal quantum numbers. Note that more than one spherical harmonics can be combined with the same radial function $P_{i}(r)$ to obtain the given orbital $\phi_{j}(\mathbf{r})$ except the case $l_{i}=0$. That is why the different subscripts $i$ and $j$ are used in $P$ and $\phi$.

The radial density takes the form

$$
\varrho(r)=\sum_{i} f_{i} \lambda_{i} \varrho_{i}(r)
$$

where $\rho_{i}(r)=P_{i}^{2}(r)$ and $\lambda_{i}$ are the radial occupation numbers. According to the Unsöld theorem, the density is strictly spherically symmetric for closed shells, that is, $\lambda_{i}=2 l_{i}+1$ [or having both spin up and down electrons $\left.\lambda_{i}=2\left(2 l_{i}+1\right)\right]$. Otherwise, the density is only approximately spherically symmetric.

Definition. Define now a generating function

$$
\sigma(r)=\sum_{i} a_{i} \varrho_{i}(r)
$$

where 


$$
a_{i}=w_{i} f_{i} \lambda_{i}
$$

The factors $w_{i}$ are selected as

$$
w_{i}=e^{-\alpha \varepsilon_{i}-\gamma l_{i}\left(l_{i}+1\right)}
$$

with any real values $\alpha$ and $\gamma$.

Theorem 1. The generating function gives the density if $\alpha=\gamma=0$.

Proof. Substituting $\alpha=\gamma=0$ in Eq. (18), all $w_{i}=1$ and Eq. (16) is the same as Eq. (15).

We can see that the generating function is an extension of the density. While the density depends on the radial distance $r$ and the inverse temperature $\beta$, the generating function has two other variables: $\alpha$ and $\gamma$.

Theorem 2. The generating function satisfies the equation

$$
\begin{aligned}
\sigma^{\prime \prime \prime}(r)= & 8 v_{K S}(r) \sigma^{\prime}(r)+4 v_{K S}^{\prime}(r) \sigma(r)+8 \frac{\partial \sigma^{\prime}(r)}{\partial \alpha} \\
& -\frac{4}{r^{2}} \frac{\partial \sigma^{\prime}(r)}{\partial \gamma}+\frac{4}{r^{3}} \frac{\partial \sigma(r)}{\partial \gamma},
\end{aligned}
$$

where ' stands for the derivation with respect to the radial coordinate $r$.

Proof. Dividing Eq. (14) by $P_{i}(r)$, differentiating, and then multiplying by $P_{i}^{2}(r)$, we are led to

$$
\begin{aligned}
-\frac{1}{2} & P_{i}(r) P_{i}(r)^{\prime \prime \prime}+\frac{1}{2} P_{i}^{\prime}(r) P_{i}^{\prime \prime}(r)-\frac{l_{i}\left(l_{i}+1\right)}{r^{3}} P_{i}^{2}(r) \\
+ & v_{K S}^{\prime}(r) P_{i}^{2}(r)=0 .
\end{aligned}
$$

Multiplying Eq. (14) by $P_{i}^{\prime}(r)$ and combining it with Eq. (20), we obtain

$$
\begin{aligned}
P_{i}(r) P_{i}^{\prime \prime \prime}(r)+3 P_{i}^{\prime}(r) P_{i}^{\prime \prime}(r)= & 2 \frac{l_{i}\left(l_{i}+1\right)}{r^{2}} \varrho_{i}^{\prime}(r)+4 v_{K S}(r) \varrho_{i}^{\prime}(r) \\
& -4 \epsilon_{i} \varrho_{i}^{\prime}(r)-2 \frac{l_{i}\left(l_{i}+1\right)}{r^{3}} \varrho_{i}(r) \\
& +2 v_{K S}^{\prime}(r) \varrho_{i}(r) .
\end{aligned}
$$

Calculate now the derivatives of the generating function,

$$
\begin{gathered}
\sigma^{\prime}(r)=\sum_{i} a_{i} \varrho_{i}^{\prime}(r), \\
\frac{\partial \sigma^{\prime}(r)}{\partial \alpha}=-\sum_{i} a_{i} \epsilon_{i} \varrho_{i}^{\prime}(r), \\
\frac{\partial \sigma(r)}{\partial \gamma}=-\sum_{i} a_{i} l_{i}\left(l_{i}+1\right) \varrho_{i}(r),
\end{gathered}
$$

and

$$
\sigma^{\prime \prime \prime}(r)=2 \sum_{i} a_{i}\left[P_{i}(r) P_{i}^{\prime \prime \prime}(r)+3 P_{i}^{\prime}(r) P_{i}^{\prime \prime}(r)\right]
$$

Combining Eqs. (20)-(25), we arrive at Eq. (19).

Theorem 3. The square root of $\sigma(r)$ satisfies the Schrödingerlike equation

$$
-\frac{1}{2}\left(\sigma^{1 / 2}(r)\right)^{\prime \prime}+\left(v_{K S}(r)+u(r)\right) \sigma^{1 / 2}(r)=0,
$$

where $u(r)$ satisfies the equation

$$
\frac{1}{2} \sigma(r) u^{\prime}(r)+\sigma^{\prime}(r) u(r)=g(r)
$$

and

$$
g(r)=\frac{\partial \sigma^{\prime}(r)}{\partial \alpha}-\frac{1}{2 r^{2}} \frac{\partial \sigma^{\prime}(r)}{\partial \gamma}+\frac{1}{2 r^{3}} \frac{\partial \sigma(r)}{\partial \gamma} .
$$

$u(r)$ can be expressed from Eq. (27) as

$$
u(r)=\frac{2}{\sigma^{2}(r)} \int_{0}^{r} \sigma\left(r_{1}\right) g\left(r_{1}\right) d r_{1} .
$$

Proof. Using the definition of $\sigma(r)$ [Eq. (16)], one can immediately calculate that

$$
-\frac{1}{2} \frac{\left(\sigma^{1 / 2}\right)^{\prime \prime}(r)}{\sigma^{1 / 2}(r)}=\frac{1}{8}\left(\frac{\sigma^{\prime}(r)}{\sigma(r)}\right)^{2}-\frac{1}{4} \frac{\sigma^{\prime \prime}(r)}{\sigma(r)} .
$$

Defining $u(r)$ as

$$
u(r)=\frac{1}{2} \frac{\left(\sigma^{1 / 2}\right)^{\prime \prime}(r)}{\sigma^{1 / 2}(r)}-v_{K S}(r),
$$

substituting it into Eq. (27) and taking into account Eq. (19), we find that $u(r)$ satisfies Eq. (27). Equation (29) shows how $u(r)$ can be expressed with $\sigma(r)$. One can easily verify it by substituting Eq. (29) into Eq. (27).

Theorem 4. The noninteracting kinetic energy can be expressed as

$$
T_{s, \beta}=\left.\left[-\int_{0}^{\infty} \frac{\partial \sigma(r)}{\partial \alpha} d r-\int_{0}^{\infty} \sigma(r) v_{K S}(r) d r\right]\right|_{\alpha=\gamma=0} .
$$

Proof. The noninteracting kinetic energy can be written as

$$
T_{s, \beta}=-\frac{1}{2} \sum_{i} f_{i} \lambda_{i} \int \phi_{i}^{*}(\mathbf{r}) \nabla^{2} \phi_{i}(\mathbf{r}) d \mathbf{r} .
$$

It can be formulated with the radial wave functions $P_{i}(r)$

$$
\begin{aligned}
T_{s, \beta} & =-\frac{1}{2} \sum_{i} f_{i} \lambda_{i} \int_{0}^{\infty}\left[P_{i}(r) P_{i}^{\prime \prime}(r)-\frac{l_{i}\left(l_{i}+1\right)}{r^{2}} P_{i}^{2}(r)\right] \\
& =\sum_{i} f_{i} \lambda_{i} \varepsilon_{i} \int_{0}^{\infty} P_{i}^{2}(r) d r-\int_{0}^{\infty} \rho(r) v_{K S}(r) d r .
\end{aligned}
$$

The Kohn-Sham equations (14) and the form of the radial density (15) are utilized. Taking advantage of Theorem 1 and Eq. (23), we arrive at Eq. (32). 


\section{DISCUSSION}

The generating function can be considered as a generalization of the density. $\sigma$ depends not only on the variables $r$ and $\beta$ but also on two extra parameters $\alpha$ and $\gamma$. This generalization makes it possible to derive a single differential equation for $\sigma$. That is, we do not have to solve the Kohn-Sham equations. Note that at nonzero temperature, the number of partially occupied orbitals is infinite in principle. It is enough to solve Eq. (19) or the Schrödinger-like equation (26) with Eq. (29). It is an advantage of the present method over Kohn-Sham calculations. The appearance of the two extra variables is, of course, unfavorable computationally. However, as our aim is to determine the density, we only need $\sigma$ and its derivatives at $\alpha=\gamma=0$. Therefore, it is enough to calculate $\sigma$ in the neighborhood of $\alpha=\gamma=0$.

The present approach is valid both for zero and nonzero temperature. It is expected to be more beneficial in the case of elevated temperature because of the huge number of Kohn-Sham orbitals.

Equation (26) is a Schrödinger-like equation for the square root of $\sigma$. It can be compared with the Euler equation (12) that can be written in spherically symmetric case as

$-\frac{1}{2}\left(\varrho^{1 / 2}\right)^{\prime \prime}(r)+\left(v_{\theta}(r)-\frac{1}{\beta} \frac{\delta S_{s}}{\delta n(\mathbf{r})}+v_{K S}(r)-\mu\right) \varrho^{1 / 2}(r)=0$.

Equation (26) at $\alpha=\gamma=0$ has the form

$$
-\frac{1}{2}\left(\varrho^{1 / 2}\right)^{\prime \prime}(r)+\left(v_{K S}(r)+u(r, \alpha=\gamma=0)\right) \varrho^{1 / 2}(r)=0,
$$

that is,

$$
u(r, \alpha=\gamma=0)=v_{\theta}(r)-\mu-\frac{1}{\beta} \frac{\delta S_{s}}{\delta n(r)} .
$$

At zero temperature, Eq. (37) reduces to $u(r, \alpha=\gamma=0)=v_{\theta}(r)-\mu$. Observe that Eq. (26) does not explicitly depend on $\mu$. It is in accord with the fact that the grand canonical potential is a function of the difference $v-\mu$.u comprises all terms except $v_{K S}$, we do not have to be concerned about $\mu$ or the term containing the functional derivative of the noninteracting entropy.

It should be emphasized that one needs the Kohn-Sham potential to obtain the solution. The Kohn-Sham potential depends on the inverse temperature, but does not depend on $\alpha$ and $\gamma$. The KohnSham potential is needed to calculate $\sigma$. In the knowledge of $\sigma$, one can immediately obtain the density. From the density, one can compute the Kohn-Sham potential. With this new Kohn-Sham potential, one can calculate again $\sigma$. This procedure should be followed until self-consistency. Observe that the accuracy of the results will depend on the approximate exchange-correlation functional used to compute the Kohn-Sham potential.

We note that the results presented in this article might be useful not only in spherically symmetric systems. For example, it can be generalized for muffin-tin orbitals of solid-state calculations. ${ }^{25}$ In the muffin-tin approximation, the nuclei are surrounded by spheres in which the KS potential is taken to be spherically symmetric. Therefore, it is likely that the present approach can be applied in the muffin-tin method. However, this is a subtle problem that should be the subject of further research.
Finally, a simple example is presented for illustration. Consider a linear harmonic oscillator with unit mass and frequency $\omega$. The potential is $v(x)=\omega^{2} x^{2} / 2$. The generating function $\sigma$ is defined as

$$
\sigma(x)=\sum_{i} f_{i} e^{-\alpha \varepsilon_{i}} \psi_{i}^{2}(x),
$$

where $\varepsilon_{i}$ and $\psi_{i}(x)$ are the eigenvalues and eigenfunctions of the harmonic oscillator. Note that there is no $\gamma$ here. For a one-dimensional system, Eq. (19) takes the form

$$
\sigma^{\prime \prime \prime}(x)=8 v \sigma^{\prime}(x)+4 v^{\prime}(x) \sigma(x)+8 \frac{\partial \sigma^{\prime}(x)}{\partial \alpha},
$$

where' stands for the derivation with respect to $x$. For the special case of high temperature, i.e., $f_{i} \approx e^{-\beta\left(\varepsilon_{i}-\mu\right)}$,

$$
\sigma(x)=e^{\beta \mu} \sum_{i} e^{-(\beta+\alpha) \varepsilon_{i}} \psi_{i}^{2}(x)
$$

has an analytical form ${ }^{26}$

$$
\begin{aligned}
\sigma(x)= & e^{\beta \mu}\left(\frac{\omega}{2 \pi \sinh [(\alpha+\beta) \omega)]}\right)^{1 / 2} \\
& \times \exp \left[-\omega x^{2}\left(\tanh \left[\frac{(\alpha+\beta) \omega}{2}\right]\right)\right] .
\end{aligned}
$$

Substitution of Eq. (41) into Eq. (39) shows that the generating function satisfies Eq. (39) (it corresponds to Theorem 2 in one dimension). The density can be easily obtained from Eq. (41) with $\alpha=0$,

$$
\rho(x)=e^{\beta \mu}\left(\frac{\omega}{2 \pi \sinh (\beta \omega))}\right)^{1 / 2} \exp \left[-\omega x^{2}\left(\tanh \left[\frac{\beta \omega}{2}\right]\right)\right] .
$$

In the knowledge of $\sigma(x)$, we can calculate the potential $u(x)$ from Eq. (31),

$$
\begin{aligned}
u(x) & =\frac{1}{2} \frac{\left(\sigma^{1 / 2}\right)^{\prime \prime}(x)}{\sigma^{1 / 2}(x)}-v(x) \\
& =-\frac{\omega^{2} x^{2}}{2\left(\cosh \left[\frac{(\alpha+\beta) \omega}{2}\right]\right)^{2}}-\frac{\omega}{2} \tanh \left[\frac{(\alpha+\beta) \omega}{2}\right] .
\end{aligned}
$$

Selecting $\alpha=0$, we arrive at

$$
u(x)=-\frac{\omega^{2} x^{2}}{2\left[\cosh \left(\frac{\beta \omega}{2}\right)\right]^{2}}-\frac{\omega}{2} \tanh \left[\frac{\beta \omega}{2}\right] .
$$

The noninteracting kinetic energy can be gained from Eq. (32),

$$
T_{s, \beta}=e^{\beta \mu} \frac{\omega}{8} \frac{\cosh \left(\frac{\beta \omega}{2}\right)}{\left[\sinh \left(\frac{\beta \omega}{2}\right)\right]^{2}} .
$$

Figure 1 presents the potential $u(x)$ for $\omega=1$ (hartree/ $\hbar)$ and some values of $\beta \omega(\beta \omega=2,3$, and 4$)$. Figure 1 shows the potential $v(x)$, too. While $v(x)$ is positive, $u(x)$ is negative for all values of $x$ in accord with Eq. (44). 


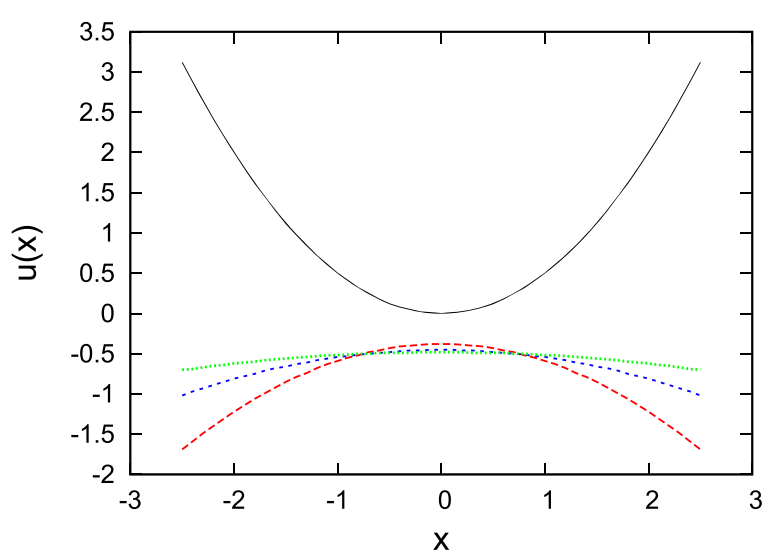

FIG. 1. Potential $v(x)$ (black solid line) and $u(x)$ as a function of $x$ for $\beta \omega=2$ (red long dashed line), $\beta \omega=3$ (blue short dashed line), and $\beta \omega=4$ (green dotted line).

In summary, an alternative orbital-free DFT approach is presented. The method does not need the kinetic energy functional. Instead, a generating function $\sigma$ is defined for spherically symmetric systems. Compared to the density, it has two extra variables and it reduces to the density if these variables are equal to zero. A thirdorder differential equation for $\sigma$ and a Schrödinger-like equation for the square root of $\sigma$ are derived. The theory is valid for spherically symmetric systems in case of zero or elevated temperature. For nonspherically symmetric systems, the method can be applied within the muffin-tin approximation.

\section{ACKNOWLEDGMENTS}

This research was supported by the National Research, Development, and Innovation Fund of Hungary, financed under the Grant No. 123988 funding scheme.

\section{REFERENCES}

${ }^{1}$ P. Hohenberg and W. Kohn, Phys. Rev. 136, B864 (1964).

${ }^{2}$ N. D. Mermin, Phys. Rev. 137, A1441 (1965).

${ }^{3}$ W. Kohn and L. J. Sham, Phys. Rev. 140, A1133 (1965).

${ }^{4}$ Y. A. Wang and E. A. Carter, in Theoretical Methods in Condensed Phase Chemistry, Progress in Theoretical Chemistry and Physics, edited by S. D. Schwartz (Kluwer, Dordrecht, 2000), p. 117.

${ }^{\mathbf{5}}$ V. L. Ligneres and E. Carter, in Handbook of Materials Modeling, edited by S. Yip (Springer, Dordrecht, 2005), p. 137.

${ }^{6}$ H. Chen and A. Zhou, Numer. Math.: Theory Methods Appl. 1, 1 (2008).

${ }^{7}$ C. Huang and E. A. Carter, Phys. Rev. B 81, 045206 (2010).

${ }^{8}$ T. A. Wesolowski, J. Phys. Chem. 97, 8050 (1993).

${ }^{9}$ I. Shin and E. A. Carter, J. Chem. Phys. 140, 18A531 (2014).

${ }^{10}$ V. V. Karasiev, R. S. Jones, S. B. Trickey, and F. E. Harris, in New Developments in Quantum Chemistry, edited by J. L. Paz and A. J. Hernández (Transworld Research Network, Kerala, India, 2009), p. 61.

${ }^{11}$ V. V. Karasiev, T. Sjotstrom, D. Chakraborty, J. D. Dufty, K. Runge, F. E. Harris, and S. B. Trickey, in Computational Challenges in Warm Dense Matter, edited by F. Graziani et al. (Springer International Publishing Switzerland, 2014).

${ }^{12}$ S. B. Trickey, V. V. Karasiev, and A. Vela, Phys. Rev. B 84, 075146 (2011).

${ }^{13}$ Á. Nagy, J. Chem. Phys. 135, 044106 (2011).

${ }^{14}$ Á. Nagy, Int. J. Quantum Chem. 110, 2117 (2010).

${ }^{15}$ Á. Nagy, Chem. Phys. Lett. 460, 343 (2008).

${ }^{16}$ Á. Nagy, Int. J. Quantum Chem. 116, 862 (2016).

${ }^{17}$ H. Levämäki, Á. Nagy, K. Kokko, and L. Vitos, Phys. Rev. A 92, 062502 (2015).

${ }^{18}$ H. Eschrig, Phys. Rev. B 82, 205120 (2010).

${ }^{19}$ A. Pribram-Jones, S. Pittalis, E. K. U. Gross, and K. Burke, in Computational Challenges in Warm Dense Matter, edited by F. Graziani et al. (Springer International Publishing Switzerland, 2014).

${ }^{20}$ M. Levy, J. P. Perdew, and V. Sahni, Phys. Rev. A 30, 2745 (1984).

${ }^{21}$ N. H. March, Phys. Lett. A 113, 66 (1985).

${ }^{22}$ N. H. March, Phys. Lett. A 113, 476 (1986).

${ }^{23}$ N. H. March, J. Comput. Chem. 8, 375 (1987).

${ }^{24}$ M. Levy and H. Ou-Yang, Phys. Rev. A 38, 625 (1988).

${ }^{25}$ L. Vitos, Computational Quantum Mechanics for Materials Engineers: The EMTO Method and Applications (Springer-Verlag, London, 2007).

${ }^{26}$ R. K. Pathria, Statistical Mechanics, 2nd ed. (Butterworth-Heinemann, Oxford, 1996), p. 114 